\title{
Recent insight into the mechanism of proton-induced composite particle emission in inclusive reactions
}

\author{
A.A. Cowley ${ }^{1,2, a}$, S.S. Dimitrova ${ }^{3}$, E.V. Zemlyanaya ${ }^{4}$, K.V. Lukyanov ${ }^{4}$, and J.J. van Zyl ${ }^{1}$ \\ ${ }^{1}$ Department of Physics, Stellenbosch University, Private Bag X1, Matieland, 7602, South Africa \\ ${ }^{2}$ iThemba Laboratory for Accelerator Based Sciences, P O Box 722, Somerset West 7129, South Africa \\ ${ }^{3}$ Institute for Nuclear Research and Nuclear Energy, Bulgarian Academy of Sciences, 1784 Sofia, Bulgaria \\ ${ }^{4}$ Joint Institute for Nuclear Research, 141980 Dubna, Russia
}

\begin{abstract}
Recent results for the inclusive reaction ${ }^{93} \mathrm{Nb}(p, \alpha)$ between incident energies of 65 and $160 \mathrm{MeV}$ are shown to be consistent with a competition between knockout and pickup. The pre-equilibrium statistical multistep process terminates in either mechanism. The incident-energy dependence of the reaction reflects the dynamics of the participating reaction components. It is consequently not surprising to observe knockout to dominate at the lower and highest incident energies, with pickup very prominent in between.
\end{abstract}

\section{Introduction}

The mechanism of the $(p, \alpha)$ reaction is of considerable interest. Gadioli et al. [1] point out that apart from a pickup process, knockout of $\alpha$-clusters should also be a prominent reaction mechanism. Although they are unable to show a difference in predicted cross section and analyzing power angular distributions for their specific target species and incident energy range, it is implied that the analyzing power could in general be a valuable observable to explore details of the mechanism. Bonetti et al. [2] demonstrate that for ${ }^{58} \mathrm{Ni}(p, \alpha)^{55} \mathrm{Co}_{\text {g.s. }}$ pickup accounts for the mechanism, whereas the inclusive reaction ${ }^{58} \mathrm{Ni}(p, \alpha)$ is clearly driven by knockout of an $\alpha$-cluster. No explanation was offered for the difference.

In comprehensive studies [3-5] of the $(p, \alpha)$ reaction to the continuum of excitation on various targets at incident energies in the range of interest from 65 to $200 \mathrm{MeV}$, with one notable exception, we identify the knockout process as terminating a statistical multistep mechanism. In these studies, analyzing power angular distributions at various emission energies are very sensitive to identify both the intranuclear statistical N-N multisteps, and also the terminating process leading to emitted $\alpha$-particles.

In our most recent work [5] we confirmed that knockout dominates in the mechanism of ${ }^{93} \mathrm{Nb}(p, \alpha)$ reaction at incident energies of 65 and $160 \mathrm{MeV}$. However, at an intermediate incident energy of $100 \mathrm{MeV}$ pickup is the most important process. In this paper we discuss this surprising behaviour and we offer an explanation for the strange incident-energy trend.

\footnotetext{
ae-mail: aac@sun.ac.za
}

\section{Typical features of inclusive reactions}

Proton-induced reactions into the continuum of excitation energies are understood reasonably well in terms of statistical multistep pre-equilibrium models, such as the Feshbach, Kerman, Koonin (FKK) formulation [6]. It is interesting that the angle-integrated yield of the $(p, \alpha)$ reaction is considerably less than for $\left(p, p^{\prime}\right)$, an example of which is shown in Fig. 1. In spite of the low relative yield, the FKK theory which closely links $(p, \alpha)$ with the $\left(p, p^{\prime}\right)$ pre-equilibrium chain gives a reasonably good account of the former reaction, as shown in Fig. 2. For illustration of typical agreement between theoretical FKK predictions and experimental results we have chosen an example in Fig. 2 for which pickup is accompanied by some knockout. However, we show only the summed cross sections and combined analyzing power values. For the magnitude of the separate contributions the reader is referred to Ref. [5].

\section{Reaction mechanism as reflected in the analyzing power angular distribution}

As was already mentioned, the analyzing power angular distribution is relatively sensitive to the reaction mechanism. In Fig. 3 distributions are shown for the reaction ${ }^{93} \mathrm{Nb}(p, \alpha)$ at incident energies of 65 and $100 \mathrm{MeV}$, at similar excitation energies. In both cases solid lines indicate the theoretical predictions which produce the best agreement with the experimental data. This means that for 65 $\mathrm{MeV}$ incident energy the solid curve applies to knockout in the final phase of the reaction, whereas this same solid line style is used for pickup at an incident energy of 100 $\mathrm{MeV}$. Clearly different mechanisms are in best agreement with the data as the incident energy increases from 65 to 


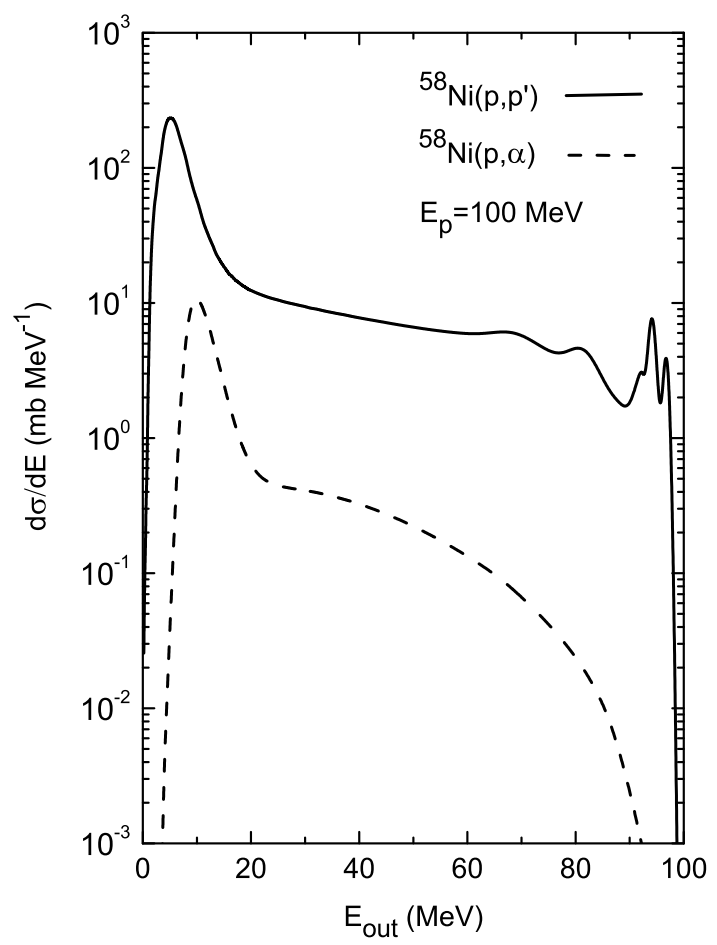

Figure 1. Angle-integrated yields for the reactions $\left(p, p^{\prime}\right)$ and $(p, \alpha)$ on ${ }^{58} \mathrm{Ni}$ at an incident energy of $100 \mathrm{MeV}$ as predicted by the code TALYS [7].

$100 \mathrm{MeV}$. It should be mentioned that at $100 \mathrm{MeV}$ incident energy there is evidence of some contribution from knockout, but it is definitely not dominant [5].

At an incident energy of $160 \mathrm{MeV}$ [4] the mechanism reverts back to being mostly knockout. Consequently we encounter a very intriguing phenomenon. At an incident energy of $65 \mathrm{MeV}$ the reaction mechanism for the inclusive reaction ${ }^{93} \mathrm{Nb}(p, \alpha)$ is driven overwhelmingly by knockout as the final multistep process. Then it changes to being mostly pickup at $100 \mathrm{MeV}$, and then finally it goes back to knockout at $160 \mathrm{MeV}$. This behaviour was observed for the first time in Ref. [5], and it needs to be interpreted in terms of the respective dynamics of knockout and pickup.

\section{Description of knockout and pickup}

Knockout and pickup mechanisms can schematically be represented as in the diagrams in Fig. 4. For knockout we assume that only the observed $\alpha$-particle escapes from the residual nuclear system. This is a reasonable assumption because we are mostly concerned with $\alpha$-particles which are emitted at forward angles in a $(p, \alpha)$ reaction. This means that associated protons will be directed towards the residual recoiling nucleus, and will thus be absorbed. This means that knockout reactions will be constrained by exactly the same momentum mismatch between the incident and exit channels as pickup under these conditions.

The crucial quantity which determines the cross section in the distorted-wave Born approximation (DWBA) is

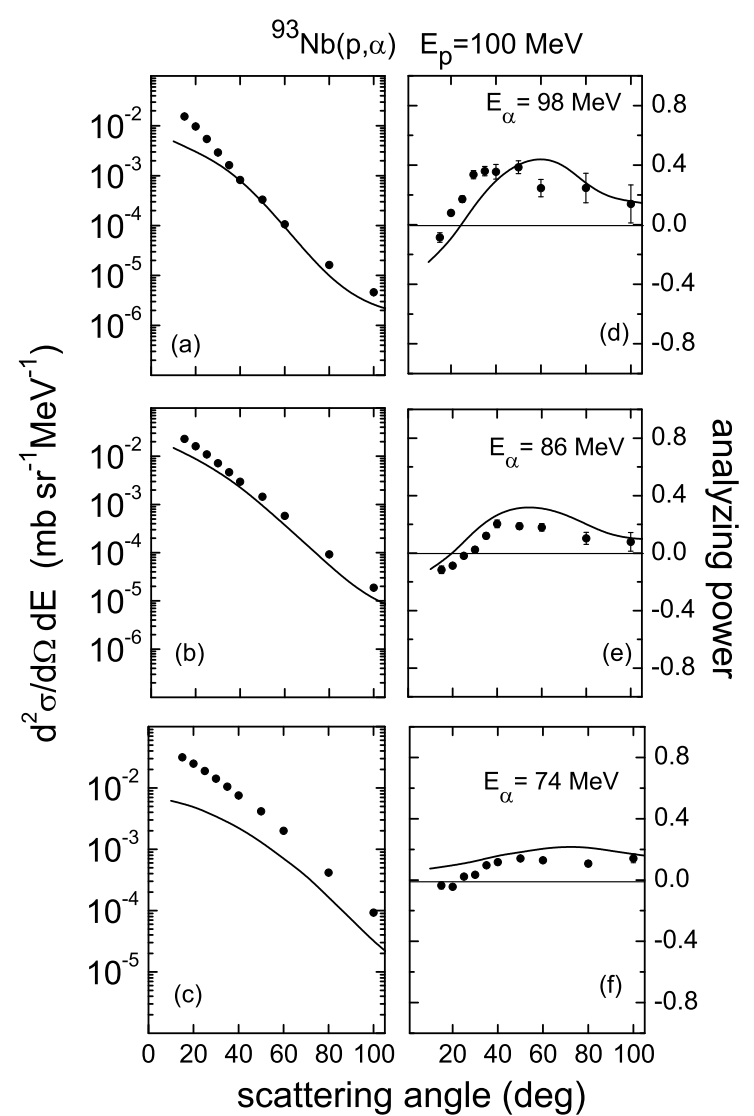

Figure 2. Double-differential cross sections (a)-(c) and analyzing power (d)-(f) as a function of scattering angle for the ${ }^{93} \mathrm{Nb}(p, \alpha)$ reaction at an incident energy of $100 \mathrm{MeV}$ and various $\alpha$-particle emission energies $E_{\alpha}$ as indicated. Theoretical curves represent the combined contributions of both pickup and knockout reaction mechanisms. Individual contributions from each reaction component are displayed in Ref. [5].

a radial integral $I$, which is given for a reaction $\mathrm{A}(a, b) \mathrm{B}$ as [8]

$$
I=\int d r_{c} \chi^{(-)\left(k_{b}, \frac{B}{A} r_{a}\right)} \phi_{C}\left(r_{c}\right) \chi^{(+)}\left(k_{a}, r_{a}\right)
$$

where $r_{a}$ and $r_{b}\left(=\frac{B}{A} r_{a}\right)$ are the relative coordinates for the system $(a \mathrm{~A})$ and $(b \mathrm{~B})$, respectively. The quantities $k_{a}$ and $k_{b}$ are the corresponding momenta of the distorted waves $\chi$. The bound state with core $\mathrm{C}$ is specified by the wave function $\phi_{C}\left(r_{c}\right)$ with relative coordinate $r_{c}$.

If we use the relationship between the coordinates as employed by Chant and Roos [9], namely

$$
\mathbf{r}_{a A}=\mathbf{r}_{a b}+\frac{B}{A} \mathbf{r}_{b B}
$$

and

$$
\mathbf{r}_{a B}=\mathbf{r}_{a b}+\mathbf{r}_{b B}
$$

before invoking separation of angular and radial coordinates, we obtain

$$
I=\int d r \chi^{(-)\left(k_{b}, \frac{B}{A} r\right)} \psi_{c}(r) \chi^{(+)\left(k_{a}, r\right)}
$$




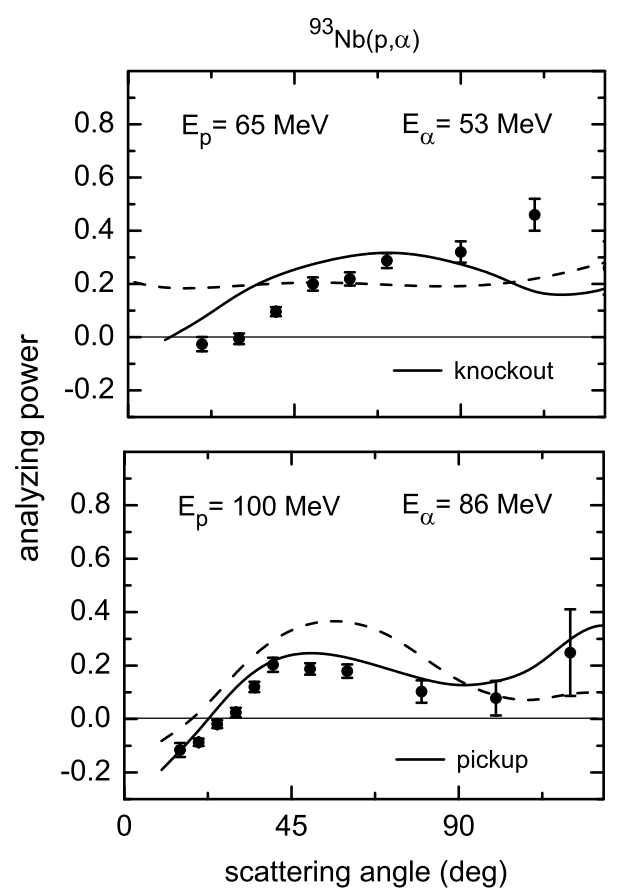

Figure 3. Analyzing power as a function of scattering angle for the ${ }^{93} \mathrm{Nb}(p, \alpha)$ reaction at incident energies of $65 \mathrm{MeV}$ (top panel) and $100 \mathrm{MeV}$ (bottom panel). The $\alpha$-particle emission energies $E_{\alpha}$ are as indicated. In both cases the outgoing energy is roughly $13 \mathrm{MeV}$ lower than the incident energy. Note that knockout is represented by the solid curve for $65 \mathrm{MeV}$, in contrast with pickup which was chosen to be displayed in this way at an incident energy of $100 \mathrm{MeV}$. In each case the respective alternative mechanism is represented as a dashed curve.

where $r=r_{a b}$ and $\psi$ is either the bound $\alpha$-cluster system (for knockout) or the three-nucleon system for pickup, approximated as a single entity in our analyses. In a plane wave approximation, and with $\mathrm{B} \approx \mathrm{A}, \mathrm{Eq}$. (4) becomes

$$
I=\int d r e^{-i\left(k_{b}-k_{a}\right) r} \psi_{c}(r) \equiv \psi_{c}\left(k_{b}-k_{a}\right) .
$$

Equation (5) means that the cross section for either knockout or pickup will be determined roughly by the momentum difference (mismatch) between the incident channel and the exit channel. The square of the absolute value of the appropriate bound state wave function in momentum space is then proportional to the cross section for each of the two mechanisms.

\section{Momentum dependence of knockout and pickup}

Clearly, as explained in Sec. 4, momentum mismatch between the incident and exit channels determines the behaviour of the cross section for both knockout and pickup, irrespective of which is the terminating mechanism in preequilibrium reactions. Consequently any difference in the signature associated with the two mechanisms would be caused mainly by the availability of angular momentum transfer in the case of pickup. Knockout involves four nucleons which preferentially share valence neutron and
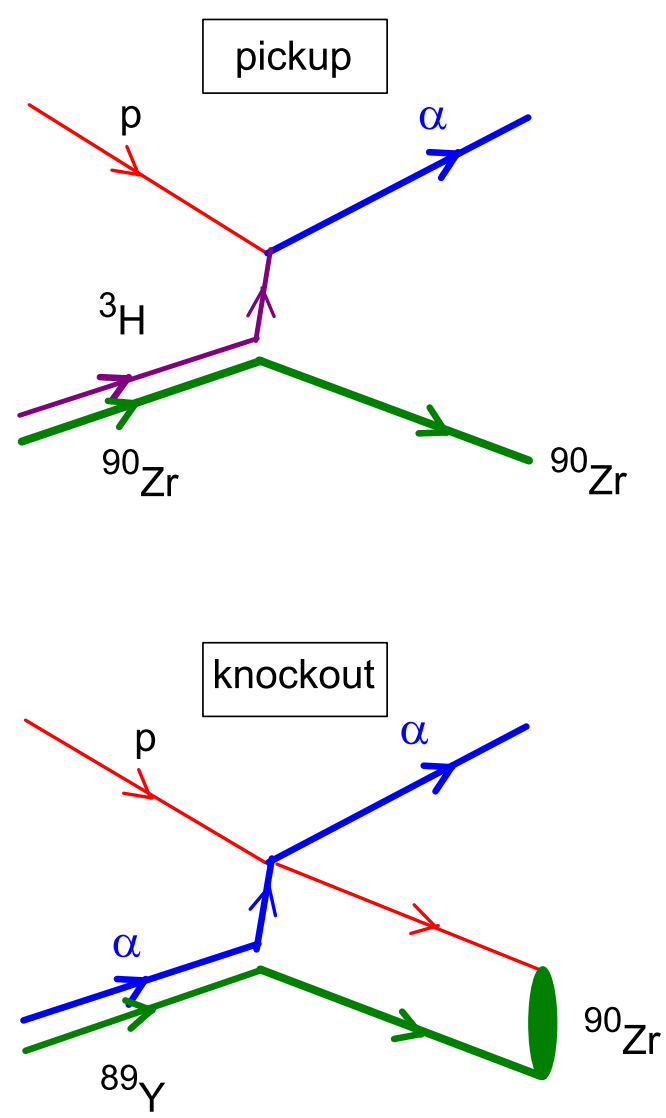

Figure 4. (Color online) Schematic representations of a pickup reaction and also a knockout mechanism. Knockout is illustrated for the case when only the struck $\alpha$-particle escapes from the residual nuclear system.

proton shells to occupy a paired system, which should have a low orbital angular momentum relative to a core. In fact, experimentally for even-even nuclei the relative angular momentum for an $\alpha$-cluster is zero [10]. On the other hand, pickup in a $(p, \alpha)$ reaction comprises two neutrons and a single proton (nominally a triton), which is not a fully paired-nucleon system. Therefore, relative to the core the combination of these three-particles has a fairly large orbital angular momentum.

In Fig. 5, a typical $\alpha$-cluster momentum distribution [11] is displayed with two ranges of momentum mismatch indicated for incident energies between 65 and $160 \mathrm{MeV}$ for the reaction ${ }^{93} \mathrm{Nb}(p, \alpha)$ at an excitation of approximately $28 \mathrm{MeV}$. The upper (hatched) range is appropriate for knockout, for which angular momentum transfer is not available. The lower (cross hatched) values become accessible for pickup, where an angular momentum transfer of $\Delta L=8$ (which corresponds to a linear momentum shift of $\Delta L / R \approx 300 \mathrm{MeV} / \mathrm{c}$ ) is required to move the effective momentum distribution to a position straddling the range around zero momentum. Of course, other values of angular momentum transfer are also allowed in a pickup reaction in transitions from the initial to various excited states in the continuum. However, due to its momentummatching property, the transfer which involves $\Delta L=8$ will contribute the dominant proportion of the cross section 


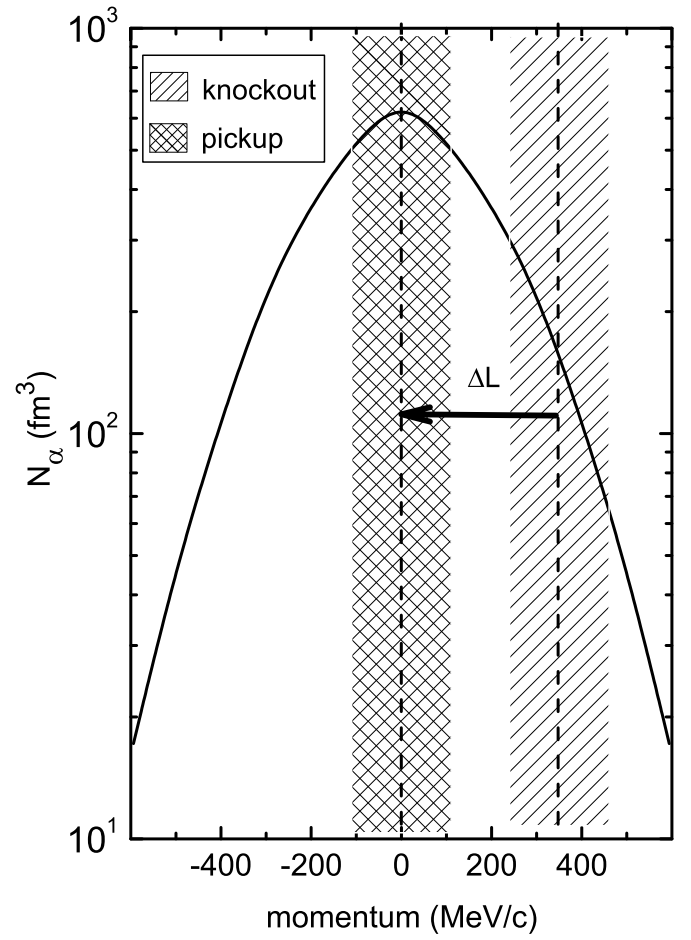

Figure 5. Momentum distribution of an $\alpha$-cluster in a typical nucleus. The momentum range of mismatch for the ${ }^{93} \mathrm{Nb}(p, \alpha)$ reaction at incident energies between 65 and $160 \mathrm{MeV}$ for an excitation of approximately $28 \mathrm{MeV}$ is shown at the upper end. Symmetrically around zero momentum of the $\alpha$-cluster distribution the range is shown as shifted by an angular momentum transfer of $\Delta L=8$ in a pickup reaction.

yield. Therefore, for the purpose of discussion, we ignore contributions from all other angular momentum transfers. In Fig. 5, for illustration, an $\alpha$-cluster momentum distribution is displayed, but a three-particle (approximated as a cluster) momentum distribution would be qualitatively similar.

This simplistic reasoning explains fully why it should not be surprising to find cross section trends for knockout and pickup which follow the lower and upper regions of momentum ranges as displayed in Fig. 5. This is exactly what is found experimentally, as is shown in Fig. 6.

In Fig. 6, extracted experimental values of the cross sections for knockout and pickup at the smallest forward scattering angle are shown at emission energies of

$$
E_{\alpha}=\left(E_{p}-20\right) \mathrm{MeV} .
$$

The specific choice of emission energy $E_{\alpha}$ is to ensure that the inclusive reaction is mostly a single-step pickup or knockout process, as is known from earlier studies. Of course, at lower emission energies, which are not used, the ejectile will be more likely to originate from intranuclear nucleon projectiles which are products of preceding multistep collisions. For those cases the interpretation of the reaction is more complicated.

At $E_{p}$ of 65 and $160 \mathrm{MeV}$, for which only a knockout process is identified in our analysis, it is assumed that pickup accounts for a below-observable threshold of about

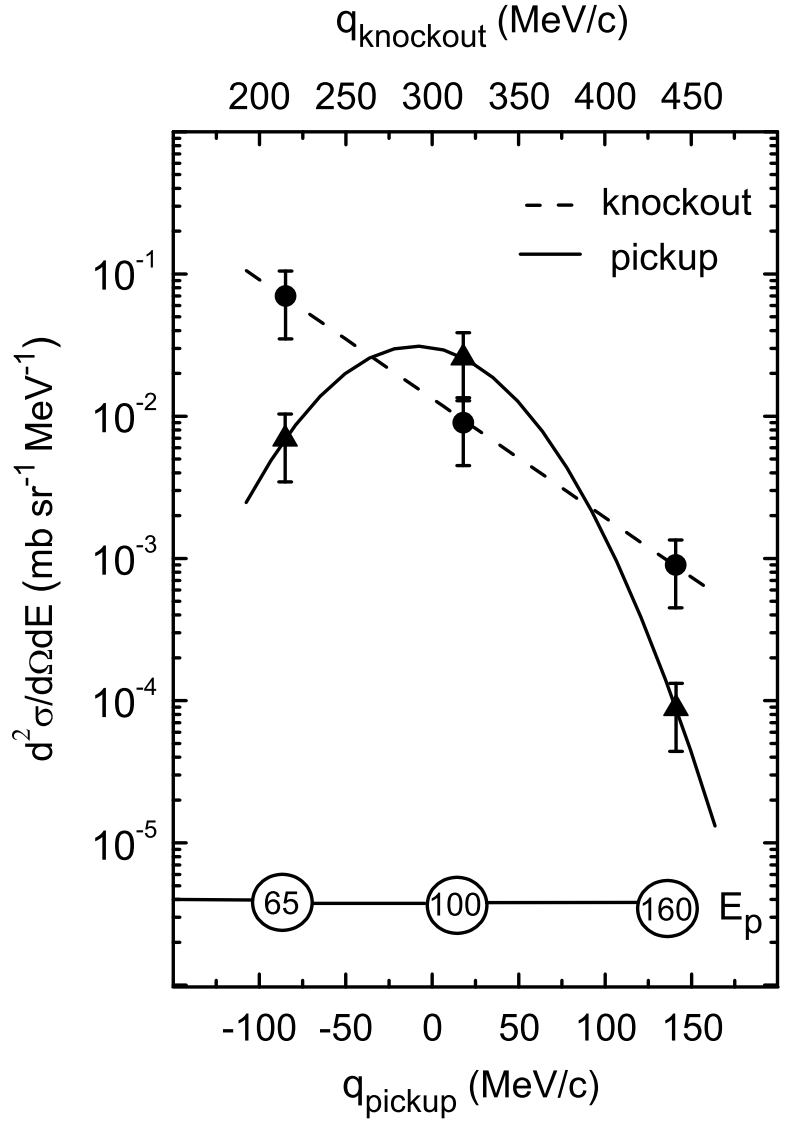

Figure 6. Experimental incident energy dependence of the cross sections extracted from Ref. [5] shown as a function of effective momentum transfer for knockout and pickup, respectively. At incident energies of 65 and $160 \mathrm{MeV}$ (lowest and highest momentum points), where knockout is dominant, a $10 \%$ pickup contribution was adopted as a reasonable limit. At an incident energy of $100 \mathrm{MeV}$ (central values) the relative magnitudes for knockout and pickup as extracted from the results reported in Ref. [5] were used. Curves are fits to the experimental points. Error bars were estimated as a reasonable uncertainty based on the accuracy of extraction of relative cross sections. The top horizontal momentum scale applies to knockout and the bottom scale is for pickup. The incident energy $E_{p}$ for each set of experimental points is indicated in the circles associated with a nonlinear energy scale above the lower horizontal axis.

$10 \%$ of the experimental values. This gives an upper limit for pickup at these energies corresponding to the upper error bar. At $100 \mathrm{MeV}$ incident energy, at the same kinematic conditions of angle and emission energy, the total experimental cross section is split between pickup and knockout in the proportion as found in the analysis to give best agreement with the cross section and analyzing power angular distributions. At this incident energy the pickup yield increases rapidly relative to the knockout portion as emission energy drops, as shown in the example of Fig. 7.

For knockout, results are plotted in Fig. 6 as a function of momentum difference between incident and exit channels. For pickup an additional shift in momentum is introduced to compensate for an angular momentum transfer of $\Delta L=8$. 
The trend of the cross sections with incident energy for knockout tracks the expected exponential decrease with momentum of an $\alpha$-cluster in a bound target system. Pickup follows a rough symmetry around an adjusted value of zero momentum. Of course, in an inclusive pickup reaction, not only a single angular momentum transfer enters. However, based on the possible orbitals of valence nucleons which could participate in the pickup process, it is not unreasonable to assume an upper limit of around $\Delta L=8$ occurs for ${ }^{93} \mathrm{Nb}$. For various reasons lower angular momentum transfers contribute progressively less to the cross section.

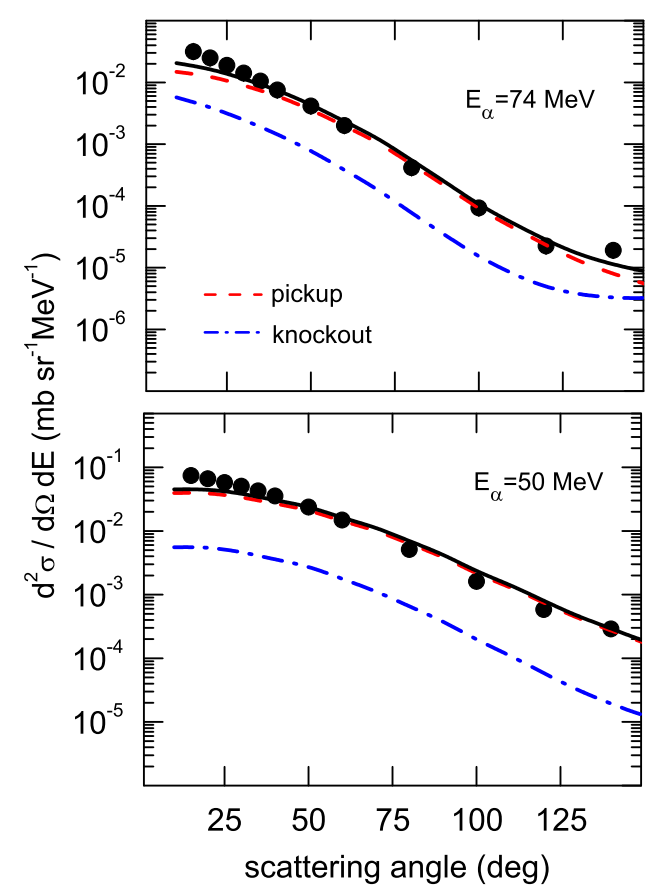

Figure 7. (Color online) Double-differential cross sections as a function of scattering angle for the ${ }^{93} \mathrm{Nb}(p, \alpha)$ reaction at an incident energy of $100 \mathrm{MeV}$ at two values of the emission energy $E_{\alpha}$ as indicated. The relative contributions of pickup (dashed curves) and knockout (dash-dot curves) are shown. The overall normalization is arbitrarily adjusted to the experimental data. See Ref. [5] for details of how the relative contributions of pickup and knockout are determined from the full set of cross section and analyzing power experimental data.

\section{Summary and conclusions}

The mechanism of the ${ }^{93} \mathrm{Nb}(p, \alpha)$ reaction was investigated between incident energies of 65 and $160 \mathrm{MeV}$. A statistical multistep process is the basic mechanism which describes the progression of cross section and analyzing power angular distributions for various emission energies accurately. The phenomenon of a competition between a terminating pickup or a knockout step in the preequilibrium chain, was found to be consistent with expectation based on the response of the two alternative components to the inherent momentum mismatch $\Delta P$ between the incident and exit channels. In the case of pickup, large angular momentum transfers $\Delta L$ are available. This causes the contribution of the yield from this mechanism to be optimized at the incident energy where $\Delta L$ compensates roughly for $\Delta P$. Consequently the yield for emitted $\alpha$ particles originating from a pickup process grows from a low incident energy, peaks at an intermediate value and then drops again towards the upper end of the rage of incident energies.

For knockout as part of the pre-equilibrium reaction, on the other hand, the cross section simply falls exponentially as required by the momentum distribution of bound $\alpha$-clusters in the target nucleus. This is because of generally low angular momentum for $\alpha$-clusters relative to the core in the bound target system.

Apart from different dynamics, relative yields from the two competing processes depend on $\alpha$-cluster preformation probabilities and spectroscopic factors, respectively. The apparent change in the mechanism of the ${ }^{93} \mathrm{Nb}(p, \alpha)$ reaction at incident energies from 65 to $160 \mathrm{MeV}$ is consequently not as strange as it would at first appear.

The general trend found in the present investigation is likely to be present in other nuclear species also, and this needs to be explored further. Of course, the postulated absence of large relative angular momentum for bound $\alpha$ clusters is an extrapolation from experimental studies restricted to even-even target nuclei. It would be desirable to confirm the validity of this assumption by means of explicit quasifree knockout studies of odd target nuclei.

\section{Acknowledgements}

The research of A.A.C. and J.J.v.Z. was funded by the National Research Foundation (NRF) of South Africa. The studies of S.S.D. were partially supported by the DFNI-T02/19 grant of the Bulgarian Science Foundation. E.V.Z. and K.V.L were supported by the Russian Foundation for Basic Research (RFBR) under Grants No. 12-01-00396a and No. 13-01-00060a. All authors gratefully acknowledge the various sources of financial support.

\section{References}

[1] E. Gadioli, E. Gadioli-Erba, P. Guazzoni, P.E. Hodgson, and L. Zetta, Z. Phys. A 318, 147 (1984)

[2] R. Bonetti, F. Crespi, and K.-I. Kubo, Nucl. Phys. A 499, 381 (1989)

[3] A.A. Cowley, G.J. Arendse, J.W. Koen, W.A. Richter, J.A. Stander, G.F. Steyn, P. Demetriou, P.E. Hodgson, and Y. Watanabe, Phys. Rev. C 54, 778 (1996)

[4] S.S. Dimitrova, A.A. Cowley, J.J. van Zyl, E.V. Zemlyanaya, and K.V. Lukyanov, Phys. Rev. C 89, 034616 (2014)

[5] S.S. Dimitrova, A.A. Cowley, E.V. Zemlyanaya, and K.V. Lukyanov, Phys. Rev. C 90, 054604 (2014)

[6] H. Feshbach, A. Kerman, and S. Koonin, Ann. Phys. (N. Y.) 125, 429 (1980)

[7] A.J. Koning, S. Hilaire, and M.C. Duijvestijn, Proceedings of the Int. Conference on Nuclear Data for Science and Technology, Nice, France, April 22-27, (2007), eds. O. Bersillon, F. Gunsing, E. Bauge, 
R. Jacqmin, and S. Leray, EDP Sciences, 2008, p. 211214.

[8] P.D. Kunz and E. Rost, in Computational Nuclear Physics, eds. K. Langanke et al, (Springer-Verlag, Berlin, 1993) Vol. 2, Chap. 5.
[9] N.S. Chant and P.G. Roos, Phys. Rev. C 15, 57 (1977) [10] T.A. Carey, P.G. Roos, N.S. Chant, A. Nadasen, and H.L. Chen, Phys. Rev. C 291273 (1984)

[11] A.N. Antonov, E.N. Nikolaev, I.Zh. Petkov, P.E. Hodgson, and G.A. Lalazissis, Bulg. J. Phys. 19, 11 (1992) 